\title{
Re: Laparoscopic Sleeve Gastrectomy Effects on Overactive Bladder Symptoms
}

\section{Giovanni Palleschi, Antonio Luigi Pastore, Mario Rizzello, Giuseppe Cavallaro, Gianfranco Silecchia, Antonio Carbon}

Sapienza University of Rome, Department of Sciences and Medico-Surgical Biotechnologies, Urology Unit, ICOT, Latina, Italy

J Surg Res 2015;196:307-312.

\section{EDITORIAL COMMENT}

Obesity increases the risk of several comorbidities. Though the relationship between a variety of urogenital complications and morbid obesity (MO) has been demonstrated, the information showing a relationship between overactive bladder (OAB) and MO is limited. Laparoscopic sleeve gastrectomy (LSG) is currently the most commonly chosen surgical procedure for obesity. Recently, Palleschi et al. assessed the prevalence of OAB in the MO population and the resolution of these symptoms after LSG. They included 120 patients ( 60 female-male) undergoing LSG in their study group. They created a control group of 120 patients (60 female-male) with the same criteria who were waiting for LSG. The patient group recorded a 3-day voiding diary (VD) and completed OAB short-form questionnaire (OABq-SF) 1 week before and 180 days after surgery. OAB diagnosis was present in $35 \%$ of the patient group and $28 \%$ of the control group. In the $\mathrm{MO}$ patient and control groups, OAB was found at higher rates in females than in males. In the patient group, a significant improvement was found by comparing $\mathrm{OABq}-\mathrm{SF}$ scores and VD parameters before and after LSG. There was no change found in the OABq-SF scores and VD parameters in the control group. Treatment of obesity with LSG provided an improvement in OAB symptoms. When the increasing rate of bariatric surgery in our country in recent times is considered, this is a very good study showing both the relationship between $M O$ and $O A B$, and the improvement in $O A B$ symptoms with obesity treatment.

Lokman İrkılata MD

\section{Re: Prevalence of Hydronephrosis in Women with Advanced Pelvic Organ Prolapse Christina E. Dancz1, Daphne Walker2, Diane Thomas1, Begüm Özel1 \\ 1 University of Southern California, Keck School of Medicine, Division of Female Pelvic Medicine and Reconstructive Surgery, Department of Obstetrics and Gynecology, Los Angeles, CA \\ 2Keck School of Medicine, University of Southern California, Division of Women's Imaging, Department of Radiology, Los Angeles, CA}

Urology 2015;86:250-254.

\section{EDITORIAL COMMENT}

In pelvic organ prolapse (POP) patients, hydronephrosis may develop and obstructive uropathy may be encountered as a result. Though the development mechanism of hydronephrosis is not fully known, the most frequently blamed mechanism is voiding dysfunction and bladder outlet obstruction (B00). This year, Dancz et al. included 180 female patients with POP in their study and determined the prevalence of hydronephrosis with POP and the clinical and urodynamic parameters relating to hydronephrosis. The study was designed as a prospective, observational cohort study researching hydronephrosis in women with advanced degrees of POP. Women with at least +1 points for $C_{1}$ Aa and Ba points on POP-Q investigation were assessed for hydronephrosis. The presence of diabetes mellitus was found to be related to hydronephrosis to a significant degree ( $8 \%$ to $21 \%, p=0.009)$. A greater degree of hydronephrosis was observed in those with high mean values of POP degree at $\mathrm{Aa}, \mathrm{Ba}, \mathrm{C}$ and $\mathrm{D}$ for anterior and apical POP ( $<<0.01)$, however, no such relationship was found for posterior POP. On multi-channel urodynamic tests, hydronephrosis patients had higher residual urine amounts, lower mean first leak volume and higher mean maximum cystometric capacity compared to patients without hydronephrosis. The prevalence of hydronephrosis among women with advanced POP was identified as 30.6\%. The authors concluded that diabetes mellitus and the degree of anterior or apical POP were related to hydronephrosis. Urodynamically, increased post voiding residue, higher cystometric capacity and lower volume at first leak were related to hydronephrosis. This study contains important data revealing the relationship between POP and hydronephrosis. 